\title{
Evolution of carbon nanotube dispersion in preparation of epoxy-based composites: From a masterbatch to a nanocomposite
}

\author{
M. Aravand*, S. V. Lomov, I. Verpoest, L. Gorbatikh \\ Department of Materials Engineering, KU Leuven, Kasteelpark Arenberg 44, B-3001 Leuven, Belgium
}

Received 1 February 2014; accepted in revised form 22 April 2014

\begin{abstract}
The state of carbon nanotube (CNT) dispersion in epoxy is likely to change in the process of composite production. In the present work CNT dispersion is characterized at different stages of nanocomposite preparation: in the original masterbatch with high CNT concentration, after masterbatch dilution, in the process of curing and in the final nanocomposite. The evaluation techniques included dynamic rheological analysis of the liquid phases, optical, environmental and charge contrast scanning electron microscopy, electrochemical impedance spectroscopy and dynamic mechanical analysis. The evolution of the CNT dispersion was assessed for two CNT/epoxy systems with distinctly different dispersion states induced by different storage time. Strong interactions between CNT clusters were revealed in the masterbatch with a longer storage time. Upon curing CNT clusters in this material formed a network-like structure. This network enhanced the elastic behaviour and specific conductivity of the resulting nanocomposite, leading to a partial electrical percolation after curing.
\end{abstract}

Keywords: nanocomposites, multiwall carbon nanotubes, dispersion state, rheometry, microscopy

\section{Introduction}

Carbon nanotubes (CNTs) have shown potential to improve the performance of fiber-reinforced composites when added in small quantities in the polymer matrix. They can influence the composite mechanical, electrical and thermal properties [1-3]. Reported improvements, however, vary significantly from one study to another and are not always reproducible. Variations in the dispersion state of nanotubes are likely to be responsible for these problems.

Agglomeration represents an important challenge when using CNTs as reinforcement in polymers and their composites. The excessive surface area of CNTs creates substantial van der Waals attraction forces, which together with the low viscosity of the matrix can give rise to the formation of relatively large agglomerates during composite production. The reinforcing efficiency of CNTs in the agglomerated form is known to be significantly lower than of individually dispersed CNTs [4, 5]. A uniform dispersion of nanotubes within the polymer matrix is, therefore, an important prerequisite for improved mechanical properties at minimal CNT contents $[6$, 7]. Additionally, the presence of CNT agglomerates in the liquid resin can impose serious issues when this resin is used to produce a fibre reinforced composite. Large clusters of nanotubes can be filtered out by the fibre reinforcement during the impregnation step or act as defects in consolidated composite [2]. However, agglomeration of the initially dispersed nanotubes is not always an undesirable phenomenon. For example, formation of an agglomerated CNTs network is beneficial and even necessary

\footnotetext{
*Corresponding author, e-mail: aravand@gmail.com

(C) BME-PT
} 
for electrical conductivity of the composite [7-10]. The results of recent study suggest that the presence of such a network in the matrix, can enhance certain mechanical properties of a fibre reinforced composite [11].

During the multi-step composite production process various factors can affect the dispersion of CNTs. These effects could not be necessarily anticipated and generalized through the characterization results of any other step. For instance, when the nanotubes are introduced to the epoxy resin at high concentrations (masterbatches), the dispersion state can be affected by storage history, processing conditions and also by curing reaction which can induce so called secondary agglomerations [9]. This may or may not lead to the formation of a three dimensional network of CNT agglomerates depending on the different factors and the initial dispersion state of the liquid resin.

Depending on the physical state of the system, various direct or indirect methods for the assessment of the CNT dispersion in epoxy based composites have been reported in the literature. Scanning and transmission electron microscopy are widely used to directly visualize CNT dispersion in the solid state [12-15]. Optical microscopy is also commonly used to observe larger CNT agglomerates both in liquid dispersions and solid nanocomposite films [9, 16-19]. However, microscopic techniques could not be efficiently employed at liquid state (especially in high concentration dispersions) and could merely provide a qualitative measure of the dispersion state over a small field of view.

Indirect methods based on static or dynamic rheological analyses could efficiently be employed to obtain quantitative measures of the nanoparticle dispersion in liquid resins [4, 19-22]. This is mainly based on the fact that the dispersion quality of the nanoparticles often affects the viscosity and viscoelastic properties of liquid suspensions in different ways. However, rheological characterizations are better to be confirmed by other techniques, such as microscopy, because reagglomeration of the nanotubes during measurement sometimes leads to erratic viscosity measurements $[19,22]$.

Conductive nature of CNTs has made it possible to use electric conductivity measurements to investigate percolation behaviour of particles with respect to the filler concentration, dispersion quality, etc., both in liquid and solid states [4, 23, 24]. Disper- sion state of CNTs controls interactions between nanotube clusters, hence affecting the electrical properties of the blend. It is generally accepted that a better CNT dispersion shows a higher electrical conductivity $[4,23]$; nevertheless, intensive agglomeration of the nanotubes may result in the formation of interconnected networks with enhanced conductivity. Schultz et al. have shown that the insulator to conductor transition in the epoxy/CNT composites could be controlled by re-arranging CNT networks at a constant nanotube concentration.

However, most of the studies concerning the application of CNTs in polymer composites either ignore the dispersion assessment or rely on certain preliminary evaluations limited to a specific stage of the manufacturing process.

The purpose of our research is to address this deficiency by assessing the evolution of the CNT dispersion state during the entire composite preparation process, in a comparative fashion. This comparison is made using a stepwise analysis which is illustrated on two extreme cases of the dispersion morphology. The chosen systems for the analysis are epoxy based resins with CNTs that are identical in terms of the type and concentration of the components. The only difference is the storage time of the masterbatch. More specifically, CNTs used in the two systems were chosen from masterbatches of the same type, which were stored for a different time. The difference in aging led to a different agglomeration morphology, enabling us to establish the stepwise dispersion characterization in a comparative manner. However, the aging mechanism, as well as the effect of masterbatch aging on CNTs dispersion, although being observed in this work, are not by themselves the focus of the present study and demands another detailed investigation.

The characterizations were conducted in four stages, i.e.: CNT/epoxy masterbatch, diluted masterbatch, transition stage (during curing) and cured nanocomposite, as schematically shown in Figure 1. Such a detailed analysis provides information on the state of the CNT dispersion at each step, explicating different properties observed in the resulting nanocomposites. At each step, key factors that can influence CNT dispersion are discussed. The main purpose of this study is to emphasize the importance of a stepwise dispersion analysis in preparation of the nanocomposite, and to introduce a series of different methods, suited for characterization at each 


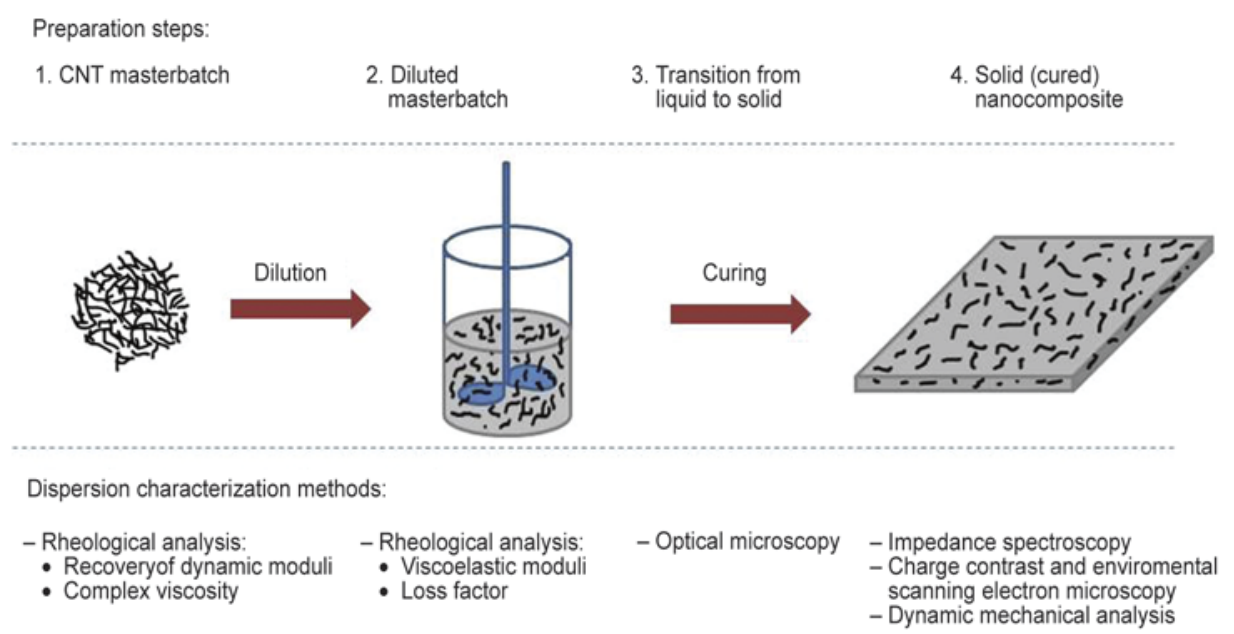

Figure 1. Schematic overview of the multistep characterization of the CNT dispersion state during preparation of CNT/epoxy nanocomposite

step. This will greatly facilitate the reproducibility of the results, which is currently one of the biggest challenges involved in manufacturing of CNT modified fibre reinforced composites.

\section{Experimental}

\subsection{Materials}

The epoxy monomer used in this work (Epikote 828LVEL) was a diglycidyl ether of bisphenol A (DGEBA). This was cured with 1,2-diaminocyclohexane (Dytek DCH-99) as hardener which was added to the resin at $15.2 \mathrm{phr}$ (parts per hundred resin). Multi-wall carbon nanotubes (MWNTs) were supplied by Nanocyl in the form of highly concentrated, pre-dispersed mixtures (masterbatches) with a similar bisphenol-A epoxy resin (Epocyl NC R128-02). MWCNTs incorporated in this masterbatch have an average diameter of around $9 \mathrm{~nm}$, a specific surface of $250-300 \mathrm{~m}^{2} / \mathrm{g}$ and a carbon purity $>90 \%$.

For the study of the CNT dispersion two CNT/epoxy model systems were prepared based on the same type of materials with equal compositions. However, CNT masterbatches used in the two systems spent different times in storage. One system is prepared from a fresh (non-aged) version of the CNT masterbatch Epocyl NC R128-02. The other system is prepared from an older (aged) version of the same masterbatch, which was received and stored almost two years before the experiments. DSC analysis of the two systems does not show any trace of curing in the aged masterbatch. Melting endotherm observed in the DSC curve of the aged masterbatch, (Figure 2) indicates that during storage, the epoxy resin in the masterbatch mixture has been partially crystallized. As it is shown, this endotherm vanishes in the second temperature sweep. However, although both systems under study are thermally treated before the experiments are carried out, it seems that partial crystallization of the epoxy resin has significantly contributed to the agglomeration of carbon nanotubes in the aged system, where CNTs are repelled from the epoxy crystallites. In the text to follow non-aged and aged systems are to be referred to as ' $\mathrm{N}$ ' and 'A', respectively. In both systems nanotube masterbatches were diluted with DGEBA epoxy so that a final CNT concentration of $0.3 \mathrm{wt} \%$ is attained. In order to homogenize the concentrated mixtures, prior to dilution, the masterbatches were thoroughly stirred using a marine type blade stirrer at $600 \mathrm{rpm}$ for 10 minutes at room temperature. The same stirring procedure was repeated following dilution of the systems and the addition of the hardener. The blends were then degassed using a vacuum

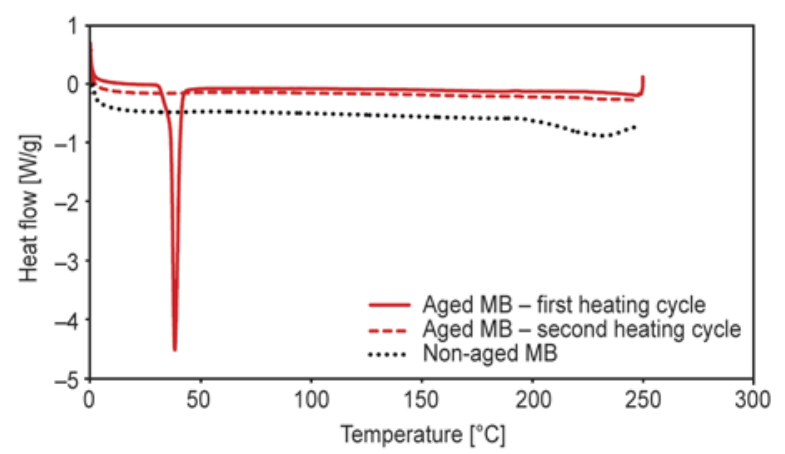

Figure 2. DSC Thermograms of the aged and non-aged masterbatches before thermal treatment, showing the first (solid line) and second (dashed line) heating cycles for the aged system 
oven for 15 minutes. Solid samples were produced by pouring and then curing the resin mixtures in aluminum molds at $70^{\circ} \mathrm{C}$ for one hour followed by post curing at $140^{\circ} \mathrm{C}$ for another one and a half hours. The produced samples were then cut into the test specimens specified further.

\subsection{Characterization methods}

Rheological measurements at stage 1 and stage 2 (see Figure 1) were carried out at room temperature using an AR2000 rheometer, equipped with a $2^{\circ}$ cone and plate geometry. The oscillation time sweep was performed at sufficiently low strain and angular frequency $(\omega)$ values of $0.5 \%$ and $0.5 \mathrm{rad} / \mathrm{sec}$, to guarantee a linear behaviour. This oscillatory test was performed in order to investigate the structure recovery behaviour of the mixture through the measurement of the time evolution of shear storage modulus $\left(G^{\prime}\right)$, right after the sample experienced a pre-shear of $100 \mathrm{~s}^{-1}$ for two minutes.

Optical microscopy was used in order to observe the nanotubes behaviour during curing at stage 3 . Imaging was carried out using an Olympus BHS Microscope. The samples of a few milligrams weight were put between two glass slides and placed in a Mettler FP82-HT hot stage. The micrographs were then obtained by a JVC TK-C1381 camera during the course of curing on time intervals of 5 seconds programmed through Qwin software from Leica Company.

Charge contrast scanning electron microscopy was carried out on the nanocomposite at stage 4 using a FEI NOVA Nanolab 600 microscope with a TLD (through the lens) detector. This was done on $5 \mu \mathrm{m}$ thick sections which were cut from the solid cured samples using a Leica Ultracut UCT microtome. No conductive layer was coated on the films so as to take advantage of the contrast effect in electron charging (or 'voltage contrast') of the sample surface. This made it possible to have an in depth view into the cured film so that the nanotubes embedded in the resin film could be observed.

Environmental Scanning Electron Microscopy (ESEM) micrographs were captured from the solid nanocomposite samples using a Philips ESEM XL30. Multiple images taken from each sample were then digitally combined into one single larger image, in order to observe a wider region. The images were taken with sufficient overlap so that the stitching is done more accurately.
The electrical properties of the nanocomposite samples were measured at stage 4 by means of a Solartron Frequency Analyzer SI 1225 together with a Solartron Electrochemical Interface SI 1287. Measurements were carried out at frequencies ranging from 0.1 to $106 \mathrm{~Hz}$ with a constant amplitude of $2 \mathrm{~V}$ and a DC potential of $0.4 \mathrm{~V}$. Samples with a dimension of $1 \mathrm{~mm} \times 10 \mathrm{~mm} \times 10 \mathrm{~mm}$ were cut and then the two surfaces of each sample were covered with a conductive silver paint.

Glass transition temperature $\left(T_{\mathrm{g}}\right)$ of the samples were identified at stage 4 using a dynamic mechanical analyzer (TA-Instruments DMA Q800 V 7.5). The analyses were carried out by heating the samples from 25 to $250^{\circ} \mathrm{C}$ with a heating rate of $3^{\circ} \mathrm{C} / \mathrm{min}$ using dual cantilever clamps at a frequency of $1 \mathrm{~Hz}$. Similar to the three point bending loading mode, it is possible to make reliable $T_{\mathrm{g}}$ measurements using a dual cantilever fixture [25]. Samples for this measurement were in the form of rectangular pieces of $3.5 \mathrm{~mm}$ thickness, $35 \mathrm{~mm}$ length and $11.5 \mathrm{~mm}$ width.

\section{Results and discussion}

\subsection{Masterbatch (stage 1)}

Highly concentrated CNT/resin mixtures (masterbatches) are nowadays widely used both at laboratory and industrial scales to facilitate the processing of nanocomposite materials [26-28]. Although the increased viscosity of the CNT masterbatch can limit diffusion and sedimentation of CNTs by restricting the Brownian motions, reduced distances between the nanotubes at high loadings can give rise to the strong attractive forces, leading to further agglomeration of CNTs during sufficiently long storage periods. Recovery of the storage and loss moduli of the two concentrated (masterbatch) systems after they were subjected to a pre-shear of a $100 \mathrm{~s}^{-1}$ for two minutes are shown in Figure 3a. Such a high pre-shear is able to destroy looser clusters of CNTs $[20,29]$.

Both systems reflect a solid-like behaviour as $G^{\prime}>G^{\prime \prime}$. Moreover, a significantly higher shear storage modulus for the aged masterbatch compared to the fresh one, observed throughout the recovery period, represents a much more pronounced elastic contribution. This indicates a stronger interaction between the nanotubes in case of the aged system where apparently a more robust network of CNT agglomerates exist. Moreover, the fresh masterbatch represents an obvious recovery over time for $G^{\prime}$ (a factor 

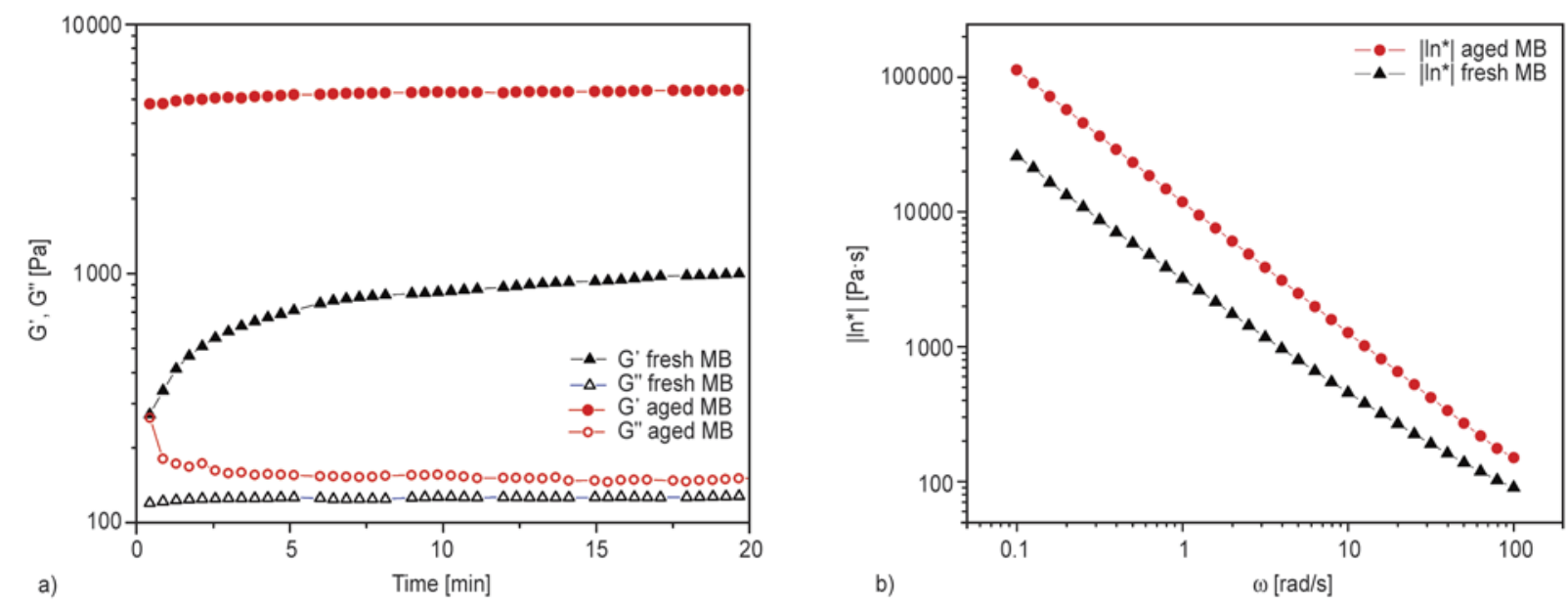

Figure 3. Rheological behaviour the aged (circles) and fresh (triangles) CNT masterbatches: a) recovery of the storage, $G^{\prime}$ (solid symbols) and loss, $G^{\prime \prime}$ (closed symbols) moduli as a function of time following a pre-shear of $100 \mathrm{~s}^{-1}$ for two minutes; b) log-log plot of the complex viscosity, $|\eta *|$, vs angular frequency

of 3), whereas in case of the aged version the corresponding recovery is smaller and faster. This suggests that CNT agglomerates (agglomerate network) existing in the aged masterbatch, are either very difficult to break under the applied pre-shear or restructure extremely fast as soon as the shearing is ceased. The complex viscosity of the aged system also shows higher values in the whole frequency range from 100 to $0.1 \mathrm{rad} / \mathrm{s}$, being more considerable at lower frequencies (Figure $3 \mathrm{~b}$ ). This indicates that the motion of the matrix molecules is more restricted in the aged masterbatch which confirms the findings in the oscillatory recover analysis.

\subsection{Diluted masterbatch (stage 2)}

The concentrated masterbatch mixtures were diluted under shear mixing to the final CNT concentration of $0.3 \mathrm{wt} \%$. This will be the ultimate concentration of nanotubes in the final composite material. It has been previously reported that the matrix preparation process can largely affect the dispersion of CNTs both before and after the curing process. For instance, Schulz et al. [9] showed that application of a high pre-shear can even enhance the agglomeration and network formation of CNTs during the following mixing steps, due to the so called shear induced nanotube agglomerate formation. Mixing time, intensity, and temperature are amongst the other important processing parameters that can influence the agglomerate formation at this stage [7, 8, 22]. In these works it has been proven that the dispersion state of the nanotubes after completion of the curing process depends greatly on their dispersion quality at the resin preparation level; Therefore, evaluation of the dispersion quality following the resin preparation step is deemed necessary, even if the initial CNT masterbatch is well mixed. In the present work we take advantage of the accuracy and sensitivity of the rheological analysis to characterize the dispersion/ agglomeration state of CNTs.

Characteristics of the microstructure and dispersion state of a suspension can be revealed by measuring the low and high frequency viscoelastic moduli [21, 22]. Figure 4 a presents linear viscoelastic storage and loss shear moduli of the diluted samples as a function of frequency.

Significant differences are observed in the viscoelastic behaviour of the two systems. Shear moduli of the 'A' system are considerably higher than that of the ' $\mathrm{N}$ ' system. Furthermore, the storage modulus $(G$ ') of the resin system 'A' (solid circles) develops a plateau at low frequencies $(\omega \rightarrow 0)$. A frequency independent rubbery plateau of $G^{\prime}$ is caused by highly interacting nanotubes, and is a well-known characteristic of a network or a structured entanglement in the suspending medium $[21,30,31]$. This, together with a loss modulus $\left(G^{\prime \prime}\right)$ remaining lower than $G^{\prime}$ all over the swept frequency range, indicates the presence of a network of entangled CNT clusters with a pseudo-solid-like behaviour. This situation is quite comparable to the cases where a solid-like gel network is emerged following an increase in the nanofiller content as observed in $[21,30]$.

In contrast, the diluted ' $\mathrm{N}$ ' resin system represents the typical hallmarks of a liquid-like material with a perfectly linear $G^{\prime \prime}(\omega)$ staying higher than $G^{\prime}$ over a wide range of frequencies. The crossover of $G^{\prime}$ and $G^{\prime \prime}$ at $50 \mathrm{rad} / \mathrm{s}$ shows that an interconnected network 

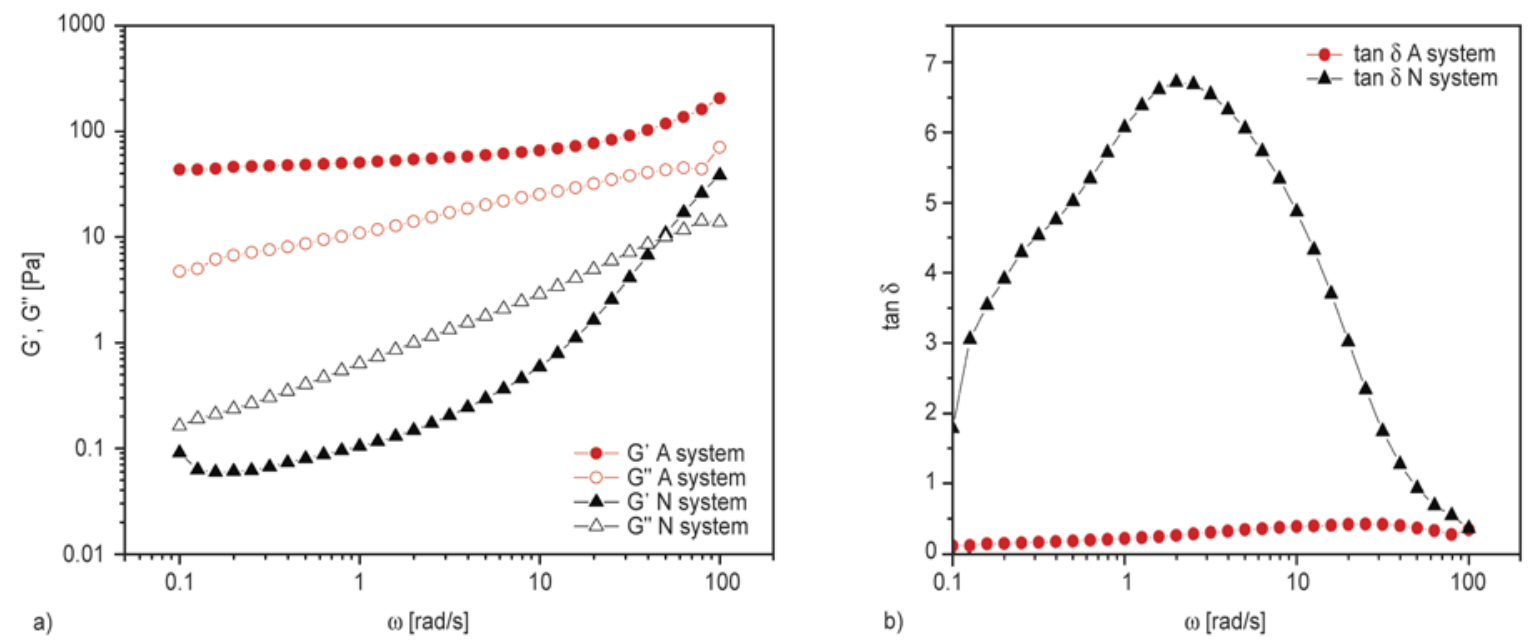

Figure 4. Rheological behaviour of the aged (circles) and non-aged (triangle) CNT (diluted to $0.3 \mathrm{wt} \% \mathrm{CNT}$ ) samples at $70^{\circ} \mathrm{C}$. a) Storage, $G^{\prime}$ (solid symbols) and loss, $G^{\prime \prime}$ (closed symbols) moduli as a function of angular frequency;

b) Loss factor, $\tan \delta$, against angular frequency

of the CNTs is formed only at higher frequencies. At this point, the initially dispersed agglomerates entangle with each other and thenceforth a dominantly elastic (rather than viscous) behaviour is observed [32].

An even more pronounced distinction between the two types of samples can be made through the evaluation of the corresponding loss factor, $\tan \delta$ $\left(=G^{\prime \prime} / G^{\prime}\right)$, as a function of frequency (Figure $4 \mathrm{~b}$ ) obtained from the dynamic moduli presented in Figure $4 \mathrm{a}$. The variations in the intensity of the peak value of $\tan \delta$ can be employed as an effective empirical measure to quantify nanotube dispersion state in a liquid matrix [22]. A better dispersed system normally exhibits a higher peak value (or $\tan \delta$ in general), which here is the case for the ' $\mathrm{N}$ ' system. This is due to the fact that the storage modulus, $G$ ', of the system with better dispersed CNTs is most often lower than that of a poorly dispersed system, as it was also the case in our system (Figure 4a) where the 'A' system exhibits a more solid-like behaviour. Lower $\tan \delta$ values observed in the 'A' system again imply its enhanced elastic properties compared to the 'N' system. Such a difference between viscoelastic properties of the two diluted systems may originate from the distinct elasticity of the entangled nanotube clusters at the masterbatch level, where stronger interactions were found in the 'A' system. Our observations at this stage indicate that the shear forces applied during the dilution step were not sufficient to homogenize the dispersion including the aged CNT masterbatch, so that a solid-like behav- iour induced by an interconnected network of CNT agglomerates still persists.

\subsection{Transition from liquid to solid (stage 3)}

Addition of the hardener and the subsequent curing reaction can both independently provoke further agglomeration (reagglomeration) of the nanotubes in an epoxy/CNT resin system $[7,16,19,33]$. This type of reagglomeration is sometimes referred to as 'secondary agglomeration' so as to differentiate it from the primary or initial agglomeration, normally existing in the dry un-processed (raw) bulk CNTs $[7,10]$. Addition of hardener can enhance CNT reagglomeration by lowering the viscosity and hence increasing the mobility of the nanotubes, even at the same temperature and before the onset of curing [16]. Furthermore, hardener can enhance ionic concentration of the dispersion, thereby leading to a reduction in the stability of the dispersion [33]. Curing temperature plays a very important role in nanoparticle reaggregation and cluster formation, as it can control the mobility of the particles by altering the matrix viscosity as well as the kinetics of the curing reaction. A trade-off exists between viscosity and curing rate as both are differently affected by temperature. A lower curing temperature will cause prolonged gelation times, so that nanoparticles (or local clusters) will have more time to organize themselves into agglomerated structures. However, this process will be slower here due to the higher viscosity of the system in comparison with curing at higher temperatures. Higher curing temperatures on 
the other hand, can create the same effect by boosting the mobility of the particles and lowering the viscosity; but instead, the onset of gelation will arrive faster. Rheological evidence discussed in the previous sections, already revealed possible presence of an interconnected network-like CNT structure with considerable elastic contribution in the ' $\mathrm{A}$ ' resin system (Figure 4). In this part of the analysis, morphological evolution of the two systems under study was monitored during the curing reaction using an optical microscope equipped with a heating element. Figure $5 \mathrm{a}-5 \mathrm{~d}$ shows the optical micro-

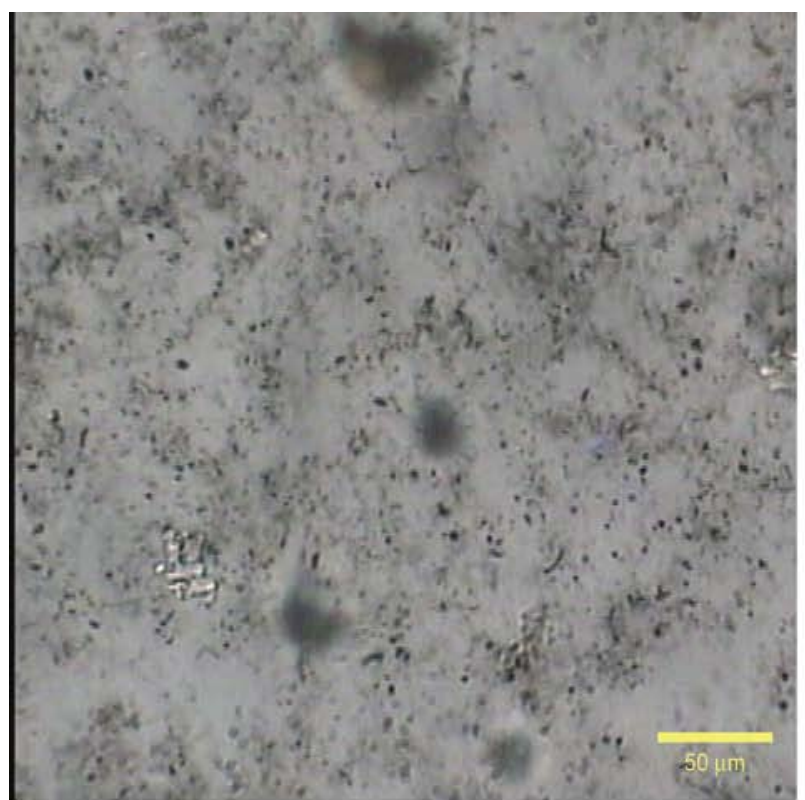

a)

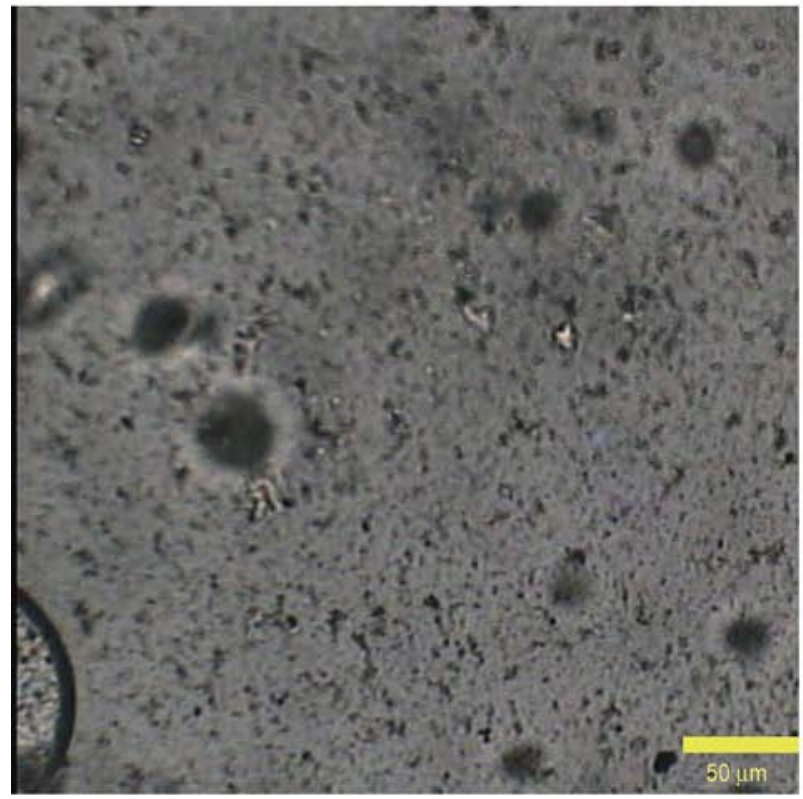

c) graphs obtained right before and after the curing reaction.

The difference between the microstructure of the two systems prior to curing (Figure 5a and 5c) is already visible. CNT clusters in the non-aged system (Figure 5c), look denser (and hence darker) while the ones in the aged system (Figure 5a) look swollen and further pushed into each other in such a way that the boundaries of the clusters are less distinguishable. This can be due to gradual resin diffusion during storage. Partial crystallization of the epoxy resin in the aged-CNT masterbatch, (Fig-

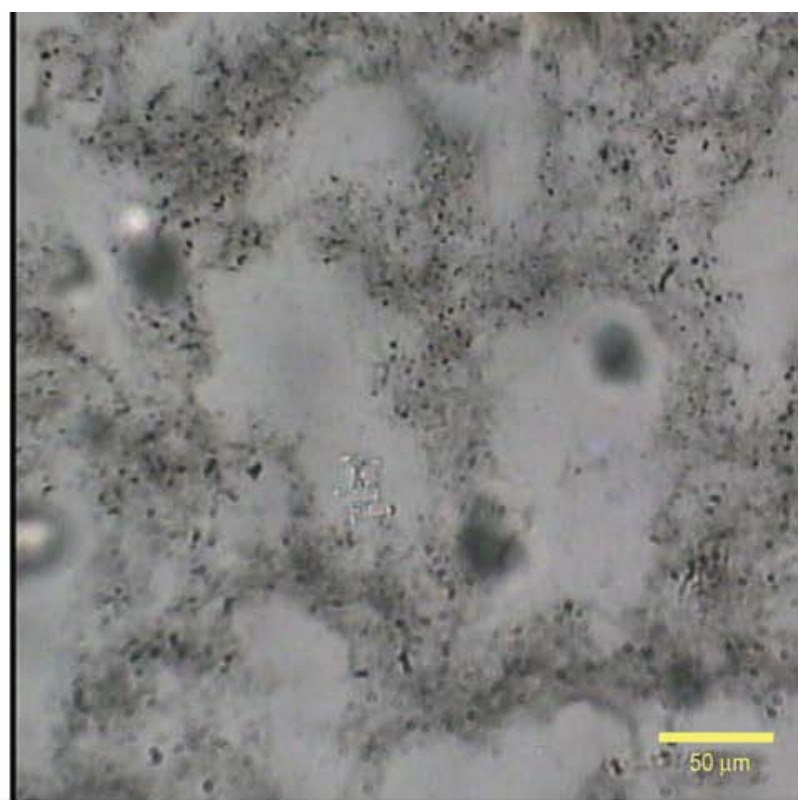

b)

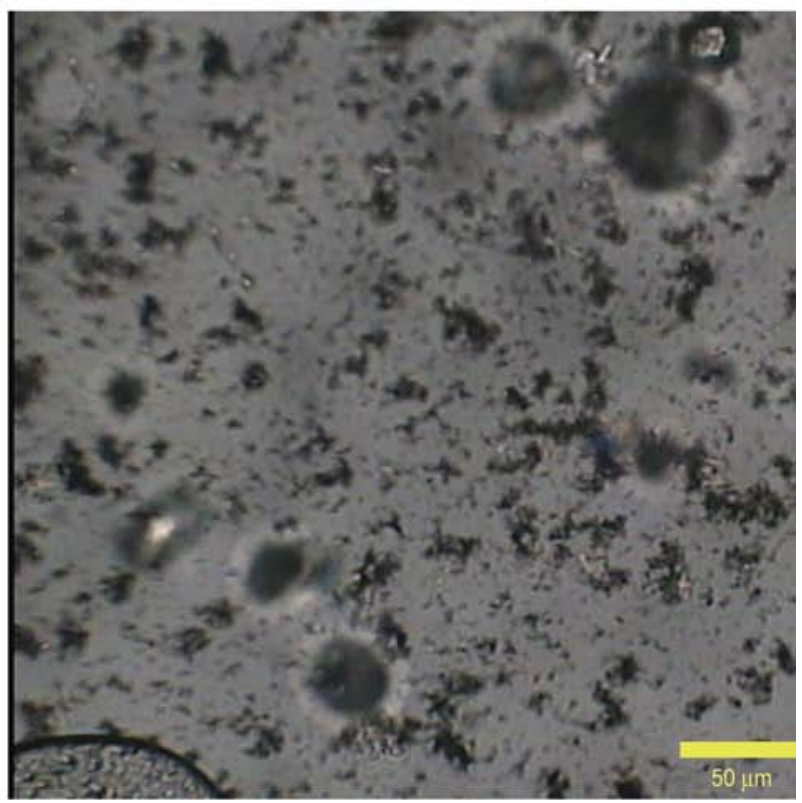

d)

Figure 5. Optical micrographs of the $0.3 \mathrm{wt} \%$ diluted CNT/epoxy suspensions during curing following the addition of hardener at $70^{\circ} \mathrm{C}$ for: system ' $\mathrm{A}$ ' before (a) and after (b) curing and system ' $\mathrm{N}$ ' before (c) and after (d) curing. Images have a $50 \mu \mathrm{m}$ scale bar 
ure 2) as discussed in section 2.1, seems to be responsible for the non-homogeneity in CNT dispersion at this stage, as can be seen in Figure 5a.

As a result of the curing reaction, the tenuous structure of the aged CNT clusters in 'A' system (Figure 5a) is transformed into a more robust three dimensional interconnected network with clear and well defined borders (Figure 5b). This is markedly different from the micro-structure of the ' $N$ ' system after curing reaction (Figure $5 \mathrm{~d}$ ). In this case, further aggregation of the clusters apparently did not lead to the formation of any macroscopic network being observed in the former case (Figure 5b). Instead, homogenously dispersed initial clusters have slightly grown into the individually distinguishable larger clusters. The higher contrast of these local clusters with the background might suggest a higher packing density of CNTs inside the agglomerates, in comparison with the lower contrast interconnected network of the 'A' system.

\subsection{Solid (cured) nanocomposite (stage 4)}

Upon completion of the curing reaction, the final microstructure of the nanotubes will be frozen by the reaction induced glass transition. Characterization of the resulting nanocomposite matrix can provide important information about the dispersion quality of CNTs in the solid state.

Due to the conductive nature of the CNTs the conductivity measurement is currently considered a popular technique for quantitative evaluation of the CNT dispersion or network formation; especially to determine the electrical percolation of the nanofillers upon increasing the filler concentration [23, 34]. Electrical percolation is identified by a sudden rise of several orders of magnitude in conductivity as a function of the volume fraction of the filler particles.

Figure 6 compares specific conductivity of the two samples, as a function of frequency.

The graphs are obtained using the modulus of the complex admittance function, calculated based on the AC impedance spectroscopy results of the samples at room temperature [23]. The sample containing aged CNT demonstrates specific conductivity values of about five orders of magnitude higher than that of the ' $\mathrm{N}$ ' sample at the lowest registered frequencies. Comparable to the results of our previous rheological analysis, where a partial mechanical percolation was identified in the 'A' system at

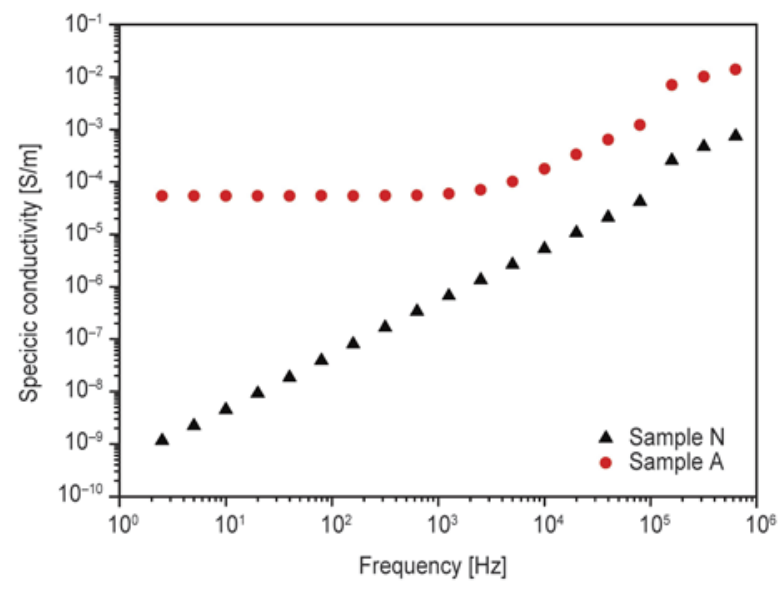

Figure 6. Log-log plot of the specific conductivity versus frequency of the cured 'A' (circles) and ' $N$ ' (squares) nanocomposites, measured at room temperature

stage 2 (diluted masterbatch, Figure 4a), here at stage 4 , the cured film of the same system reveals a frequency independent specific conductivity at lower frequencies $\left(<10^{3} \mathrm{~Hz}\right)$. This is a typical response of a percolated system in which conductive fillers created electrically connected pathways throughout the sample's thickness [23, 24, 33]. However, the frequency dependent increase in the specific conductivity observed at higher frequencies implies that this system is not fully percolated [24]. On the other hand, specific conductivity of the ' $\mathrm{N}$ ' system monotonically increases with frequency. Such a linear frequency dependent increase in conductivity is a characteristic of highly resistive materials. In fact the slope of unity observed on the log-log plot for this sample, can be explained by the expression $\sigma=$ $\omega \varepsilon^{\prime} \varepsilon_{0}$ which is used for dielectric materials [23] (where $\sigma$ : conductivity, $\varepsilon^{\prime}$ : real part of the dielectric constant, $\omega$ : angular frequency and $\varepsilon_{0}$ : vacuum permittivity). This is in fair agreement with our previous microscopic observations where formation of a network like morphology in the 'A'system was verified.

Charge contrast SEM images shown in Figure 7a and $7 \mathrm{~b}$ can provide better visualization of the nanotubes within the epoxy matrix.

In this technique the inherent difference between the charging potential of the conductive CNTs and the dielectric matrix will give rise to a charging contrast [12, 20,35]. Using a right detector (as explained in the experimental section), such a contrast will make it possible to gain some insights into the placement of the nanoparticles embedded in the matrix. Clusters of highly entangled nanotubes could read- 


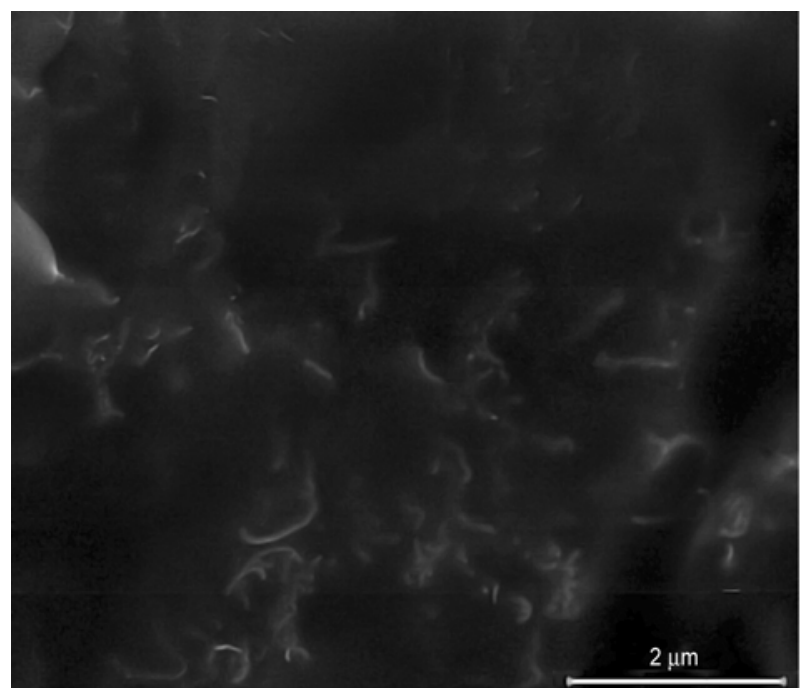

a)

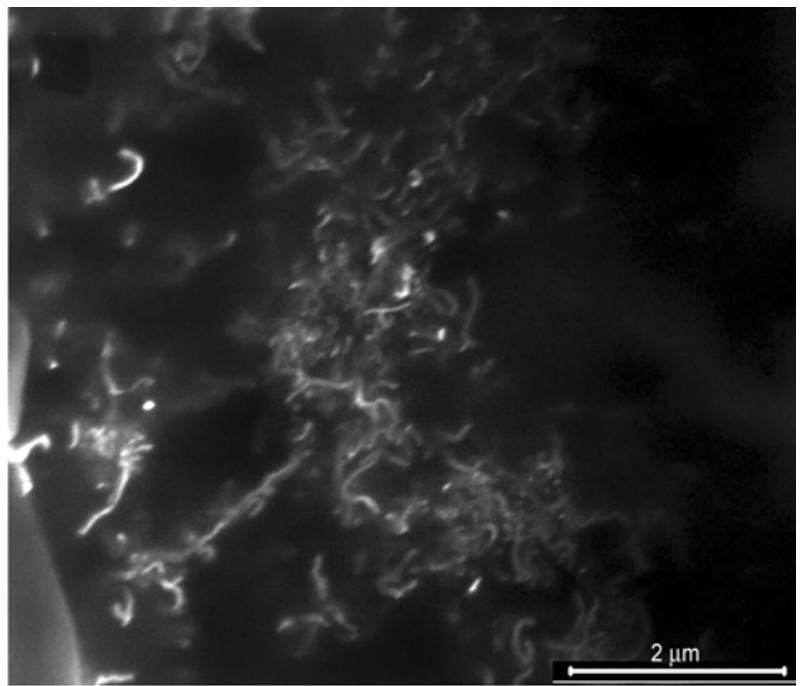

Figure 7. Charge contrast SEM micrographs of the cured a) ' $N$ ' and b) 'A' nanocomposites. Both images were taken at $35000 \times$ magnification and have a $2 \mu \mathrm{m}$ scale bar

ily be identified in these micrographs. The 'A' system distinguishes itself from the other system by larger localized clusters while a more homogenous state of dispersion can be observed in the ' $\mathrm{N}$ ' system.

Dispersion morphology of CNTs in the two systems, could be compared at a larger scale using multiple ESEM images stitched together in Figure 8a and $8 \mathrm{~b}$. Although unlike the charge contrast SEM, here the CNTs embedded in the matrix are not completely visible; one still can clearly observe large agglomerates of CNTs in the 'A' system (bright clusters), while this is not the case in the ' $N$ ' system, where isolated CNT aggregates are far apart.

Thermo-mechanical properties of the solid matrix materials were measured by DMA over a range of

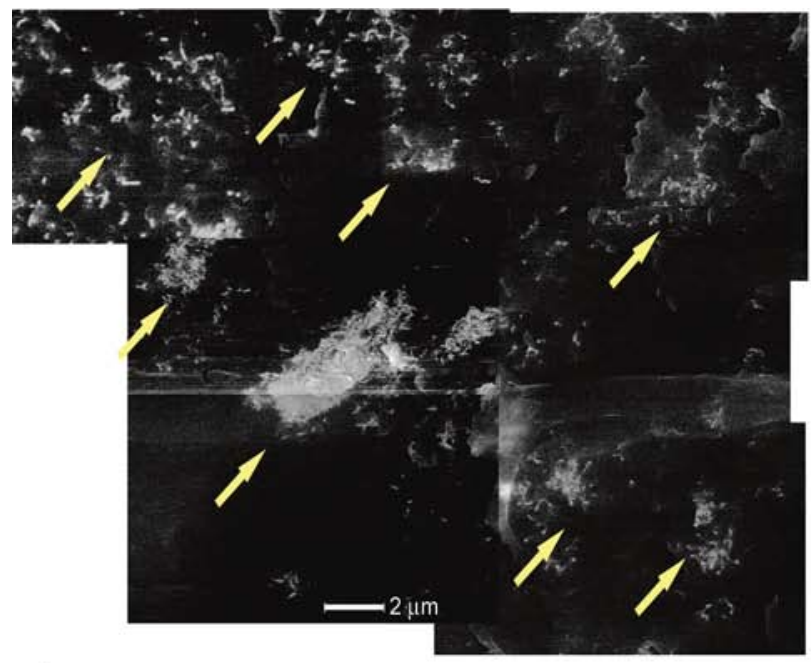

a) temperature. Figure 9 shows damping coefficient, $\tan \delta$, of the epoxy/CNT nanocomposites as well as the neat sample as a function of temperature. The major peak position of $\tan \delta$, which corresponds to the alpha relaxation or the glass transition temperature, $T_{\mathrm{g}}$, shows the highest value for the neat epoxy resin. $T_{\mathrm{g}}$ value of the 'A' system is only slightly lower than that of the neat epoxy while in case of the ' $\mathrm{N}$ ' system a considerable decrease of about $26^{\circ} \mathrm{C}$ is observed.

Effect of CNTs on the glass transition temperature of epoxy resins is controversial and a concrete conclusion based on the available data could hardly be made. Allaoui and El Bouina [36], have summarized some of the available results in a review paper.

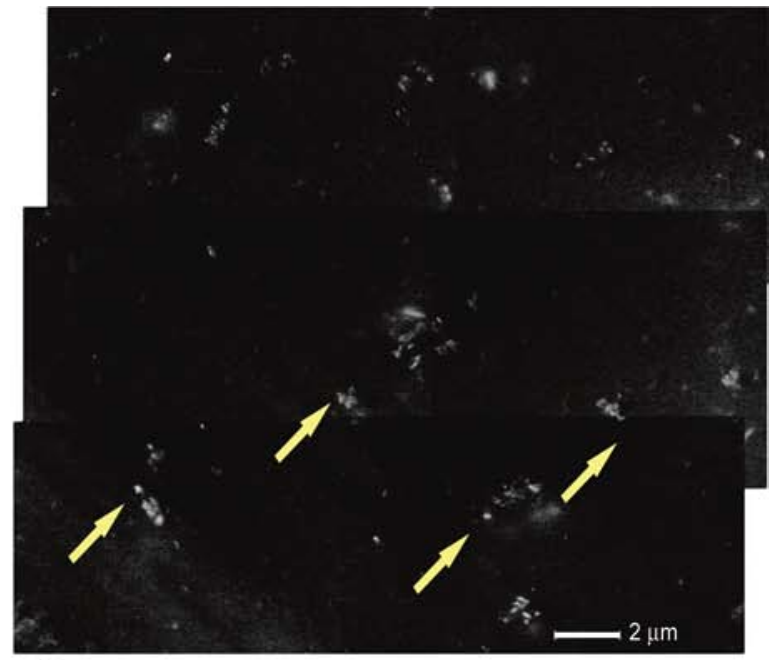

Figure 8. Stitched ESEM micrographs of the cured a) 'A' and b) 'N' nanocomposites. All images were taken at $10000 \times$ magnification and have a $2 \mu \mathrm{m}$ scale bar. Arrows indicate $\mathrm{CNT}$ agglomerates. 


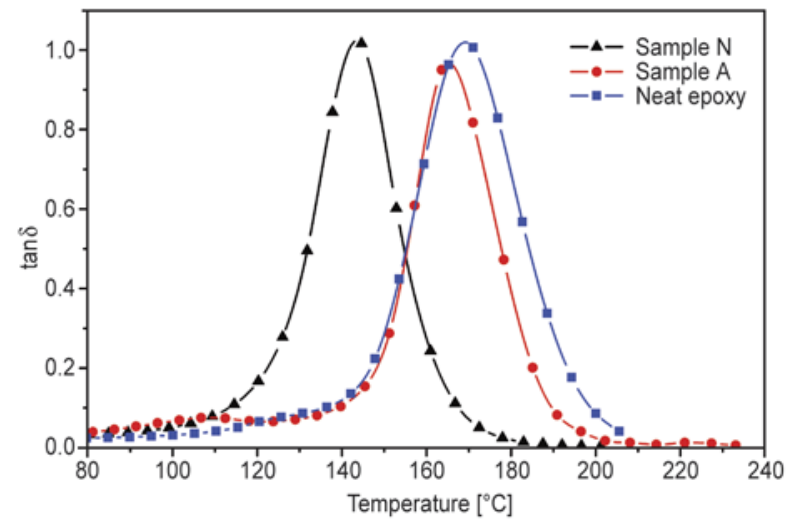

Figure 9. Tan $\delta$ versus temperature of the cured neat epoxy (squares) and ' $\mathrm{A}$ ' (circles) and ' $\mathrm{N}$ ' (triangles) nanocomposites, obtained by DMA at a frequency of $1 \mathrm{~Hz}$

Most of the reported results suggest an increase or almost no change in the $T_{\mathrm{g}}$ values $[16,19,37-41]$ while some reductions have also been reported [42, 43]. Such inconsistent results are attributed to a complex simultaneous influence of various parameters such as aspect ratio, functionality, packing density and purity (presence of metallic catalysts) of the nanotubes. Type and stoichiometric ratio of epoxy/ hardener system as well as rheological properties of the CNT containing resin are also of great importance. Specific composite processing and curing condition can control the fate of the composite material in favor of some of these competing factors.

It can be elucidated from Figure 9 that a better dispersion of the CNTs results in a lower $T_{\mathrm{g}}$ which seems to be caused by a reduced curing degree of the ' $N$ ' system. A possible explanation is that the catalytic effect of CNTs on curing [43, 44] expedites the second stage of the epoxy curing reaction which is dominantly diffusion-controlled. This results in a lower crosslinking degree and hence a lower $T_{\mathrm{g}}$. It is also likely that individual CNTs block the reaction sites in the epoxy monomer; consequently, a better dispersed system more dominantly hinders the curing reaction. This will have the same effect on the crosslinking degree as well as the glass transition temperature. This can result in a lower crosslinking degree, thus reducing the $T_{\mathrm{g}}$ of the cured epoxy. Kim et al. [45] even took advantage of the CNTs hindering effect on epoxy curing as a means of assessing the state of dispersion.

There is no doubt that dispersion evaluation techniques are not limited to those employed in this study; however, it was impossible (and perhaps not necessary) to cover all of the techniques reported in the literature in a single report. Instead, these methods were chosen in view to collect the most important information regarding the dispersion state of CNTs in each step. Moreover, the assessment methods employed at each step of the work, could be performed in more detail provided that more in depth information is desired. In such cases, interested reader is referred to the relevant references provided in each section of this study.

\section{Conclusions}

1. A methodology for the multi-step characterization of the CNT dispersion state at different stages of the nanocomposite preparation was developed. It enabled us to investigate the evolution of the CNT dispersion in the initial masterbatch, diluted masterbatch, during transition to the solid (cured) state and in the final cured nanocomposite.

2. This methodology was applied to two CNT containing epoxy based systems with extreme cases of dispersion morphologies. These different dispersion morphologies were induced by storing the CNT masterbatches for a different time.

3. Characterizations performed on the two systems revealed that fresh CNT masterbatch exhibits a transition from a solid-like to a dominantly liquid-like behaviour upon dilution, as expected; while the aged masterbatch keeps behaving dominantly solid-like after dilution. Following curing reaction, the aged system showed orders of magnitude higher electrical conductivity owing to the partial percolation of CNTs together with a higher $T_{\mathrm{g}}$ in comparison with the non-aged system.

4. The underlying cause for such distinct behaviour was found to originate from very different structures of the two systems. The existence of a highly interacting microstructure in the aged system was evident from the early stages of the stepwise characterization. This was later on evolved into a clearly visible network of CNT agglomerates during curing reaction. The footsteps of this network were identified in the postcuring solid state, where an electrical percolation induced by this network was detected. A correlation between the electrical conductivity measurements of the cured material and rheological analysis of the liquid resin was then elucidated. The results obtained in different steps of the dispersion characterization 
were always consistent and in good agreement with the morphological observations.

5. Various factors are able to independently control and alter the dispersion state of nanotubes during the nanocomposite manufacturing process. This work emphasizes the necessity of a stepwise evaluation of the dispersion quality so as to enhance reproducibility in manufacturing $\mathrm{CNT} / \mathrm{epoxy}$ nanocomposites with specific physical, thermal, electrical and mechanical properties.

\section{Acknowledgements}

The work was performed in the scope of the GOA/10/004 project 'New model-based concepts for nanoengineered polymer composites', funded by the Research Council of KU Leuven. Collaboration between KULeuven and Nanocyl S.A was performed under the framework agreement between the Department MTM, KULeuven and Nanocyl S.A. Collaboration with the Department of Chemistry and the Department of Chemical Engineering (CIT Lab.) within the framework of the Materials Research Center at KU Leuven is gratefully acknowledged.

\section{References}

[1] Joshi S. C., Dikshit V.: Enhancing interlaminar fracture characteristics of woven CFRP prepreg composites through CNT dispersion. Journal of Composite Materials, 46, 665-675 (2012).

DOI: $10.1177 / 0021998311410472$

[2] da Costa E. F. R., Skordos A. A., Partridge I. K., Rezai A.: RTM processing and electrical performance of carbon nanotube modified epoxy/fibre composites. Composites Part A: Applied Science and Manufacturing, 43, 593-602 (2012).

DOI: 10.1016/j.compositesa.2011.12.019

[3] Domingues D., Logakis E., Skordos A. A.: The use of an electric field in the preparation of glass fibre/epoxy composites containing carbon nanotubes. Carbon, 50, 2493-2503 (2012).

DOI: $10.1016 /$ j.carbon.2012.01.072

[4] Song Y. S., Youn J. R.: Influence of dispersion states of carbon nanotubes on physical properties of epoxy nanocomposites. Carbon, 43, 1378-1385 (2005).

DOI: $10.1016 /$ j.carbon.2005.01.007

[5] Gkikas G., Barkoula N-M., Paipetis A. S.: Effect of dispersion conditions on the thermo-mechanical and toughness properties of multi walled carbon nanotubes-reinforced epoxy. Composites Part B: Engineering, 43, 2697-2705 (2012).

DOI: $10.1016 /$ j.compositesb.2012.01.070

[6] Coleman J. N., Khan U., Blau W. J., Gun'ko Y. K.: Small but strong: A review of the mechanical properties of carbon nanotube-polymer composites. Carbon, 44, 1624-1652 (2006).

DOI: $10.1016 /$ j.carbon.2006.02.038
[7] Alig I., Pötschke P., Lellinger D., Skipa T., Pegel S., Kasaliwal G. R., Villmow T.: Establishment, morphology and properties of carbon nanotube networks in polymer melts. Polymer, 53, 4-28 (2012).

DOI: $10.1016 /$ j.polymer.2011.10.063

[8] Schulz S. C., Faiella G., Buschhorn S. T., Prado L. A. S. A., Giordano M., Schulte K., Bauhofer W.: Combined electrical and rheological properties of shear induced multiwall carbon nanotube agglomerates in epoxy suspensions. European Polymer Journal, 47, 2069-2077 (2011).

DOI: $10.1016 /$ j.eurpolymj.2011.07.022

[9] Schulz S. C., Schlutter J., Bauhofer W.: Influence of initial high shearing on electrical and rheological properties and formation of percolating agglomerates for MWCNT/epoxy suspensions. Macromolecular Materials and Engineering, 295, 613-617 (2010).

DOI: $10.1002 /$ mame.201000065

[10] Alig I., Skipa T., Lellinger D., Pötschke P.: Destruction and formation of a carbon nanotube network in polymer melts: Rheology and conductivity spectroscopy. Polymer, 49, 3524-3532 (2008).

DOI: $10.1016 /$ j.polymer.2008.05.037

[11] Siegfried M., Tola C., Claes M., Lomov S. V., Verpoest I., Gorbatikh L.: Impact and residual after impact properties of carbon fiber/epoxy composites modified with carbon nanotubes. Composite Structures, 111, 488-496 (2014).

DOI: 10.1016/j.compstruct.2014.01.035

[12] Kovács J. Z., Andresen K., Pauls J. R., Garcia C. P., Schossig M., Schulte K., Bauhofer W.: Analyzing the quality of carbon nanotube dispersions in polymers using scanning electron microscopy. Carbon, 45, 12791288 (2007).

DOI: $10.1016 /$ j.carbon.2007.01.012

[13] Schlea M. R., Brown T. R., Bush J. R., Criss Jr J. M., Mintz E. A., Shofner M. L.: Dispersion control and characterization in multiwalled carbon nanotube and phenylethynyl-terminated imide composites. Composites Science and Technology, 70, 822-828 (2010).

DOI: 10.1016/j.compscitech.2010.01.019

[14] Li W., Bauhofer W.: Imaging of CNTs in a polymer matrix at low accelerating voltages using a SEM. Carbon, 49, 3891-3898 (2011). DOI: 10.1016/j.carbon.2011.05.027

[15] Luo Z. P., Koo J. H.: Quantitative study of the dispersion degree in carbon nanofiber/polymer and carbon nanotube/polymer nanocomposites. Materials Letters, 62, 3493-3496 (2008). DOI: $10.1016 /$ j.matlet.2008.03.010

[16] Chakraborty A., Plyhm T., Barbezat M., Necola A., Terrasi G. P.: Carbon nanotube (CNT)-epoxy nanocomposites: A systematic investigation of CNT dispersion. Journal of Nanoparticle Research, 13, 6493-6506 (2011). DOI: $10.1007 / \mathrm{s} 11051-011-0552-3$ 
[17] Ma P-C., Mo S-Y., Tang B-Z., Kim J-K.: Dispersion, interfacial interaction and re-agglomeration of functionalized carbon nanotubes in epoxy composites. Carbon, 48, 1824-1834 (2010). DOI: $10.1016 /$ j.carbon.2010.01.028

[18] Li J., Ma P. C., Chow W. S., To C. K., Tang B. Z., Kim J-K.: Correlations between percolation threshold, dispersion state, and aspect ratio of carbon nanotubes. Advanced Functional Materials, 17, 3207-3215 (2007). DOI: $10.1002 / \mathrm{adfm} .200700065$

[19] Rahatekar S. S., Koziol K. K. K., Butler S. A., Elliott J. A., Shaffer M. S. P., Mackley M. R., Windle A. H.: Optical microstructure and viscosity enhancement for an epoxy resin matrix containing multiwall carbon nanotubes. Journal of Rheology, 50, 599-610 (2006).

DOI: $10.1122 / 1.2221699$

[20] Battisti A., Skordos A. A., Partridge I. K.: Monitoring dispersion of carbon nanotubes in a thermosetting polyester resin. Composites Science and Technology, 69, 1516-1520 (2009).

DOI: $10.1016 /$ j.compscitech.2008.05.012

[21] Galindo-Rosales F. J., Moldenaers P., Vermant J.: Assessment of the dispersion quality in polymer nanocomposites by rheological methods. Macromolecular Materials and Engineering, 296, 331-340 (2011).

DOI: 10.1002/mame.201000345

[22] Huang Y. Y., Ahir S. V., Terentjev E. M.: Dispersion rheology of carbon nanotubes in a polymer matrix. Physical Review B, 73, 125422/1-125422/9 (2006). DOI: 10.1103/PhysRevB.73.125422

[23] Sandler J. K. W., Kirk J. E., Kinloch I. A., Shaffer M. S. P., Windle A. H.: Ultra-low electrical percolation threshold in carbon-nanotube-epoxy composites. Polymer, 44, 5893-5899 (2003).

DOI: 10.1016/S0032-3861(03)00539-1

[24] Thakre P. R., Bisrat Y., Lagoudas D. C.: Electrical and mechanical properties of carbon nanotube-epoxy nanocomposites. Journal of Applied Polymer Science, 116, 191-202 (2010).

DOI: $10.1002 /$ app.31122

[25] Deng S., Hou M., Ye L.: Temperature-dependent elastic moduli of epoxies measured by DMA and their correlations to mechanical testing data. Polymer Testing, 26, 803-813 (2007). DOI: $10.1016 /$ j.polymertesting.2007.05.003

[26] Mičušík M., Omastová M., Krupa I., Prokeš J., Pissis P., Logakis E., Pandis C., Pötschke P., Pionteck J.: A comparative study on the electrical and mechanical behaviour of multi-walled carbon nanotube composites prepared by diluting a masterbatch with various types of polypropylenes. Journal of Applied Polymer Science, 113, 2536-2551 (2009).

DOI: $10.1002 / \mathrm{app} .30418$
[27] De Greef N., Gorbatikh L., Godara A., Mezzo L., Lomov S. V., Verpoest I.: The effect of carbon nanotubes on the damage development in carbon fiber/ epoxy composites. Carbon, 49, 4650-4664 (2011). DOI: $10.1016 /$ j.carbon.2011.06.047

[28] Wichmann M. H. G., Sumfleth J., Fiedler B., Gojny F. H., Schulte K.: Multiwall carbon nanotube/epoxy composites produced by a masterbatch process. Mechanics of Composite Materials, 42, 395-406 (2006).

DOI: $10.1007 / \mathrm{s} 11029-006-0050-3$

[29] Bauhofer W., Schulz S. C., Eken A. E., Skipa T., Lellinger D., Alig I., Tozzi E. J., Klingenberg D. J.: Shearcontrolled electrical conductivity of carbon nanotubes networks suspended in low and high molecular weight liquids. Polymer, 51, 5024-8027 (2010).

DOI: $10.1016 /$ j.polymer.2010.09.013

[30] Chapartegui M., Markaide N., Florez S., Elizetxea C., Fernandez M., Santamaría A.: Specific rheological and electrical features of carbon nanotube dispersions in an epoxy matrix. Composites Science and Technology, 70, 879-884 (2010). DOI: $10.1016 /$ j.compscitech.2010.02.008

[31] Trappe V., Weitz D. A.: Scaling of the viscoelasticity of weakly attractive particles. Physical Review Letters, 85, 449-452 (2000).

DOI: $10.1103 /$ PhysRevLett.85.449

[32] Fan Z., Advani S. G.: Rheology of multiwall carbon nanotube suspensions. Journal of Rheology, 51, 585604 (2007).

DOI: $10.1122 / 1.2736424$

[33] Martin C. A., Sandler J. K. W., Shaffer M. S. P., Schwarz M-K., Bauhofer W., Schulte K., Windle A. H.: Formation of percolating networks in multi-wall carbon-nanotube-epoxy composites. Composites Science and Technology, 64, 2309-2316 (2004).

DOI: 10.1016/j.compscitech.2004.01.025

[34] Moisala A., Li Q., Kinloch I. A., Windle A. H.: Thermal and electrical conductivity of single- and multiwalled carbon nanotube-epoxy composites. Composites Science and Technology, 66, 1285-1288 (2006). DOI: 10.1016/j.compscitech.2005.10.016

[35] Loos J., Alexeev A., Grossiord N., Koning C. E., Regev O.: Visualization of single-wall carbon nanotube (SWNT) networks in conductive polystyrene nanocomposites by charge contrast imaging. Ultramicroscopy, 104, 160-167 (2005). DOI: $10.1016 /$ j.ultramic.2005.03.007

[36] Allaoui A., El Bounia N-E.: How carbon nanotubes affect the cure kinetics and glass transition temperature of their epoxy composites? - A review. Express Polymer Letters, 3, 588-594 (2009). DOI: 10.3144 /expresspolymlett.2009.73 
[37] Guadagno L., De Vivo B., Di Bartolomeo A., Lamberti P., Sorrentino A., Tucci V., Vertuccio L., Vittoria V.: Effect of functionalization on the thermo-mechanical and electrical behavior of multi-wall carbon nanotube/ epoxy composites. Carbon, 49, 1919-1930 (2011). DOI: 10.1016/j.carbon.2011.01.017

[38] Guadagno L., Vertuccio L., Sorrentino A., Raimondo M., Naddeo C., Vittoria V., Iannuzzo G., Calvi E., Russo S.: Mechanical and barrier properties of epoxy resin filled with multi-walled carbon nanotubes. Carbon, 47, 2419-2430 (2009).

DOI: $10.1016 /$ j.carbon.2009.04.035

[39] Jin F-L., Ma C-J., Park S-J.: Thermal and mechanical interfacial properties of epoxy composites based on functionalized carbon nanotubes. Materials Science and Engineering: A, 528, 8517-8522 (2011).

DOI: $10.1016 /$ j.msea.2011.08.054

[40] Prolongo S. G., Gude M. R., Ureña A.: The curing process of epoxy/amino-functionalized MWCNTs: Calorimetry, molecular modelling, and electron microscopy. Journal of Nanotechnology, 2010, 420432/1420432/11 (2010).

DOI: $10.1155 / 2010 / 420432$
[41] Hsieh T. H., Kinloch A. J., Taylor A. C., Kinloch I. A.: The effect of carbon nanotubes on the fracture toughness and fatigue performance of a thermosetting epoxy polymer. Journal of Materials Science, 46, 7525-7535 (2011). DOI: $10.1007 / \mathrm{s} 10853-011-5724-0$

[42] Tao K., Yang S., Grunlan J. C., Kim Y-S., Dang B., Deng Y., Thomas R. L., Wilson B. L., Wei X.: Effects of carbon nanotube fillers on the curing processes of epoxy resin-based composites. Journal of Applied Polymer Science, 102, 5248-5254 (2006).

DOI: 10.1002 app. 24773

[43] Zhou T., Wang X., Liu X., Xiong D.: Influence of multiwalled carbon nanotubes on the cure behavior of epoxyimidazole system. Carbon, 47, 1112-1118 (2009). DOI: $10.1016 /$ j.carbon.2008.12.039

[44] Siddiqui N. A., Khan S. U., Ma P. C., Li C. Y., Kim J-K.: Manufacturing and characterization of carbon fibre/ epoxy composite prepregs containing carbon nanotubes. Composites Part A: Applied Science and Manufacturing, 42, 1412-1420 (2011). DOI: $10.1016 /$ j.compositesa.2011.06.005

[45] Kim S. H., Lee W. I., Park J. M.: Assessment of dispersion in carbon nanotube reinforced composites using differential scanning calorimetry. Carbon, 47, 26992703 (2009).

DOI: $10.1016 /$ j.carbon.2009.05.026 